\title{
FUNCTIONS WHICH REPRESENT PRIME NUMBERS
}

\section{IVAN NIVEN}

W. H. Mills [1 $]^{1}$ has proved that there is a real number $A$ so that $\left[A^{3^{n}}\right]$ is a prime for every positive integer $n$. L. Kuipers [2] has extended this and proved that for any positive integer $c \geqq 3$ there is a real number $A=A(c)$ so that

$\left[A^{\mathrm{en}}\right]$ is a prime of every positive integer $n$.

We generalize this to the following result.

THEOREM. Given any real number $c>8 / 3$, there exists a corresponding real number $A$ with property (1). Furthermore, given any real number $A>1$, there exists a value $c$ with property (1).

The proof of the first part of the theorem is a slight rearrangement of Mills' proof, and the proof of the second part employs the same basic idea in a different setting.

We begin by noting that if $x>1$ and $y \geqq 2$, then

$$
(1+x)^{y}>x^{y}+x^{y-1}+1 \text {. }
$$

Also we use, as did Mills and Kuipers, the well known result of Ingham [3] that there is a constant $K$ such that for $x \geqq 1$ there is a prime $p$ satisfying

$$
x<p<x+K x^{5 / 8} .
$$

Proof of Part 1. Write $c=8(1+d) / 3$, and choose any prime $p_{1}>K^{1 / d}$. For $n=1,2,3, \cdots$ choose the primes $p_{n+1}$ to satisfy

$$
p_{n}^{c}<p_{n+1}<p_{n}^{c}+K p_{n}^{b c / 8} .
$$

Now $K p_{n}^{5 c / 8}<p_{1}^{d} \cdot p_{n}^{5 c / 8}<p_{n}^{d+5 c / 8}=p_{n}^{c-1}$, and using (2) we can rewrite (4) as

$$
p_{n}^{0}<p_{n+1}<p_{n}^{0}+p_{n}^{c-1}<\left(1+p_{n}\right)^{c}-1 .
$$

Now this in turn implies

$$
p_{n}^{\sigma^{-n}}<p_{n+1}^{a-n-1}<\left(1+p_{n+1}\right)^{c-n-1}<\left(1+p_{n}\right)^{c^{-n}},
$$

so that $p_{n}^{c^{-n}}$ is a bounded monotonic increasing sequence, whose limit

Presented to the Society, April 28, 1951; received by the editors November 13, 1950.

${ }^{1}$ Numbers in brackets refer to the references at the end of the paper. 
we denote by $A$. Thus (6) implies

$$
p_{n}^{c-n}<A<\left(1+p_{n}\right)^{c^{-n}} \text { or } p_{n}<A^{c^{n}}<1+p_{n} \text {. }
$$

Proof of PART 2. Starting again, we now choose the prime $p_{1}$ to satisfy $p_{1}>A^{8}$ and $p_{1}>K$, and we shall prove that we can find an infinite sequence of primes $p_{2}, p_{3}, \cdots$ to satisfy the recursive inequality

$$
A^{\left\{\log p_{n}\right\}^{(n+1) / n}}<A^{\log p_{n+1}}<A^{\left(\log \left(1+p_{n}\right)\right\}^{(n+1) / n}}-1,
$$

all logarithms being to base $A$. This can be written in the form

$$
p_{n}^{\left\{\log p_{n}\right\}^{1 / n}}<p_{n+1}<\left(1+p_{n}\right)^{\left\{\log \left(1+p_{n}\right)\right\}^{1 / n}}-1 \text {. }
$$

We use induction to prove that these primes can be so chosen. Thus we shall assume that $p_{2}, p_{3}, \cdots, p_{n}$ have been obtained, and noting that the first part of inequality (8) can be written as

$$
\left(\log p_{n}\right)^{1 / n}<\left(\log p_{n+1}\right)^{1 /(n+1)},
$$

we have the inequality $\log p_{n}>\left(\log p_{1}\right)^{n}$ from our induction hypothesis. Hence the following inequalities can be written:

$$
\begin{gathered}
\left\{\log \left(1+p_{n}\right)\right\}^{1 / n}>\left\{\log p_{n}\right\}^{1 / n}>\log p_{1}>8, \\
p_{n}^{\left(\log \left(1+p_{n}\right)^{1 / n} / 8\right.}>p_{n}>p_{1}>K .
\end{gathered}
$$

Using (2), (10), and (11) we have

$$
\begin{aligned}
& \left(1+p_{n}\right)^{\left\{\log \left(1+p_{n}\right)\right\}^{1 / n}}-1>p_{n}^{\left\{\log \left(1+p_{n}\right)\right\}^{1 / n}}+p_{n}^{\left\{\log \left(1+p_{n}\right)\right\}^{1 / n}-1} \\
& >p_{n}^{\left(\log p_{n}\right)^{1 / n}}+p_{n}^{7\left(\log \left(1+p_{n}\right)\right)^{1 / n} / 8} \\
& >p_{n}^{\left\{\log p_{n}\right\}^{1 / n}}+K p_{n}^{5\left(\log p_{n}\right\}^{1 / n} / 8} \text {. }
\end{aligned}
$$

This establishes, in the light of (3), the existence of the sequence of primes satisfying (9) or (8), and we next observe that (8) implies

$$
\begin{aligned}
\left\{\log p_{n}\right\}^{1 / n} & <\left\{\log p_{n+1}\right\}^{1 /(n+1)}<\left\{\log \left(1+p_{n+1}\right)\right\}^{1 /(n+1)} \\
& <\left\{\log \left(1+p_{n}\right)\right\}^{1 / n} .
\end{aligned}
$$

Thus the bounded monotonic increasing sequence $\left\{\log p_{n}\right\}^{1 / n}$ tends to a limit which we denote by $c$, so that

$$
\left\{\log p_{n}\right\}^{1 / n}<c<\left\{\log \left(1+p_{n}\right)\right\}^{1 / n} \text { or } p_{n}<A^{c^{n}}<1+p_{n} \text {. }
$$

Thus the theorem is proved, and it can be observed that there are infinitely many values of $A$ for each value of $c$, and vice versa, because of the freedom in the choice of $p_{1}$. 


\section{References}

1. W. H. Mills, A prime-representing function, Bull. Amer. Math. Soc. vol. 53 (1947) p. 604.

2. L. Kuipers, Prime-representing functions, Neder. Akad. Wetensch. vol. 53 (1950) pp. 309-310.

3. A. E. Ingham, On the difference between consecutive primes, Quart. J. Math. Oxford Ser. vol. 8 (1937) pp. 255-266.

UNIVERSITY OF OREGON 